Bull. Austral. Math. Soc.

VOL. 66 (2002) [291-300]

\title{
GRAMIAN ANALYSIS OF MULTIVARIATE FRAME MULTIRESOLUTION ANALYSES
}

\author{
JAE KUN LIM
}

\begin{abstract}
We perform a Gramian analysis of a frame multiresolution analysis to give a condition for it to admit a minimal wavelet set and to show that the frame bounds of the natural generator for the wavelet space of a degenerate frame multiresolution analysis shrink.
\end{abstract}

\section{INTRODUCTION}

The concept of multiresolution analyses was introduced by Mallat and Meyer $[14,15]$ in order to understand and construct wavelet orthonormal (or Riesz) bases; and Benedetto and $\mathrm{Li}$ [1] generalised it to that of frame multiresolution analyses with an intention to apply it to an analysis of narrow band signals. We refer $[7,8]$ for the definitions and the properties of a Riesz basis and a frame of a Hilbert space. The close connexion between multiresolution analyses and the theory of (multi-integer) shift-invariant spaces of $L^{2}\left(\mathbb{R}^{d}\right)[5,6,9,10]$ was pointed out by several authors (see $[3,11,12,13$, for example). Especially, de Boor, DeVore and Ron [3] presented a natural generator for the wavelet space when the integer translates of a scaling function form a Riesz basis for the initial space of a multiresolution analysis (see the next section for the relevant definitions). When we consider, however, a frame multiresolution analysis in which the integer translates of a scaling function form a frame for the initial space, their generator may not be adequate. For the frame multiresolution analysis does not admit a minimal wavelet set if it is degenerate in a sense which will be explained in Section 3.

In this paper, we find a condition for a frame multiresolution analysis to admit a minimal wavelet set (Theorem 5), thereby generalising the results in $[2,13]$, and present a natural candidate for a generator of the wavelet space of a given frame multiresolution analysis. We also analyse the generator by applying the Gramian analysis of $[3,16]$ to show that the frame bounds of the generator shrink (Corollary 7).

The rest of the paper is organised as follows. Preliminary discussions are given in the next section. The statements and the proofs of the main results are presented in Section 3.

Received 6th March, 2002

Copyright Clearance Centre, Inc. Serial-fee code: 0004-9727/02 \$A2.00+0.00. 


\section{Preliminaries}

First, let us introduce a multivariate generalisation of the frame multiresolution analysis in [1].

Definition 1: $\left(\left\{V_{k}\right\}_{k \in \mathbf{Z}}, \varphi\right)$ is said to be a frame multiresolution analysis of $L^{2}\left(\mathbb{R}^{d}\right)$ if each $V_{k}$ is a closed subspace of $L^{2}\left(\mathbb{R}^{d}\right)$ and $\varphi \in V_{0}$ such that:

(1) $V_{k} \subset V_{k+1}, \quad k \in \mathbb{Z}$;

(2) $\overline{\bigcup_{k \in \mathbf{Z}} V_{k}}=L^{2}\left(\mathbb{R}^{d}\right), \bigcap_{k \in \mathbf{Z}} V_{k}=\{0\}$;

(3) $\quad D\left(V_{k}\right)=V_{k+1}, \quad k \in \mathbb{Z}$;

(4) $\left\{T_{\alpha} \varphi: \alpha \in \mathbb{Z}^{d}\right\}$ is a frame for $V_{0}$,

where $D: L^{2}\left(\mathbb{R}^{d}\right) \rightarrow L^{2}\left(\mathbb{R}^{d}\right)$ is the unitary dyadic dilation operator defined via $D f(x):=2^{d / 2} f(2 x)$, and $T_{y}: L^{2}\left(\mathbb{R}^{d}\right) \rightarrow L^{2}\left(\mathbb{R}^{d}\right)$ the unitary translation operator defined via $T_{y} f(x):=f(x-y), y \in \mathbb{R}^{d}$.

The function $\varphi$ is called a scaling function of the frame multiresolution analysis. Let $W_{0}:=V_{1} \ominus V_{0}$. If there exists a wavelet set $\left\{\psi_{1}, \psi_{2}, \cdots, \psi_{n}\right\} \subset W_{0}$ such that $\left\{T_{\alpha} \psi_{j}: \alpha \in \mathbb{Z}^{d}, 1 \leqslant j \leqslant n\right\}$ is a frame for $W_{0}$ with frame bounds $A$ and $B$, then it is easy to see that $\left\{D^{k} T_{\alpha} \psi_{j}: k \in \mathbb{Z}, \alpha \in \mathbb{Z}^{d}, 1 \leqslant j \leqslant n\right\}$ is a frame for $L^{2}\left(\mathbb{R}^{d}\right)$ with the same frame bounds $A$ and $B$ simply because $L^{2}\left(\mathbb{R}^{d}\right)=\bigoplus_{k \in \mathbf{Z}} D^{k}\left(W_{0}\right)$ by Conditions (2) and (3) of Definition 1.

In this paper, we apply the Gramian analysis developed in $[3,16]$ to investigate the inter-relationship between the spaces $V_{0}, W_{0}$ and $V_{1}$. Our analysis is based on the theory of shift-invariant spaces developed in $[3,4,5,6,9,10,16]$ which we briefly review. A closed subspace $S \subset L^{2}\left(\mathbb{R}^{d}\right)$ is said to be shift-invariant if $T_{\alpha} f \in S$ whenever $f \in S$ and $\alpha \in \mathbb{Z}^{d}$. If $\Phi \subset L^{2}\left(\mathbb{R}^{d}\right)$, then $S:=\mathcal{S}(\Phi):=\overline{\operatorname{span}}\left\{T_{\alpha} \varphi: \alpha \in \mathbb{Z}, \varphi \in \Phi\right\}$ is clearly shiftinvariant. In this case, $\Phi$ is said to be a generator of $S$. The length of $S$ is defined to be $\min \left\{\# \Phi: S=\mathcal{S}(\Phi), \Phi \subset L^{2}\left(\mathbb{R}^{d}\right)\right\}$, where \# denotes the cardinality. We use the following form of the Fourier transform: for $f \in L^{1}\left(\mathbb{R}^{d}\right) \cap L^{2}\left(\mathbb{R}^{d}\right), \hat{f}(t):=\int_{\mathbb{R}^{d}} f(x) e^{-2 \pi i t \cdot x} d x$, where - denotes the standard inner product on $\mathbb{R}^{d}$; and it is extended to be a unitary operator on $L^{2}\left(\mathbb{R}^{d}\right)$ via the Plancherel theorem. For $f \in L^{2}\left(\mathbb{R}^{d}\right), x \in \mathbb{R}^{d}$, let $\widehat{f}_{\| x}:=(\widehat{f}(x-\alpha))_{\alpha \in \mathbf{Z}^{d}}$, which is in $\ell^{2}\left(\mathbb{Z}^{d}\right)$ almost every $x \in \mathbb{T}^{d}:=[-1 / 2,1 / 2]^{d}$. For $f, g \in L^{2}\left(\mathbb{R}^{d}\right), x \in \mathbb{T}^{d}$, let

$$
[\widehat{f}, \hat{g}](x):=\left\langle\widehat{f}_{\| x}, \hat{g}_{\| x}\right\rangle \ell^{2}\left(\mathbb{Z}^{d}\right)=\sum_{\alpha \in Z^{d}} \widehat{f}(x-\alpha) \overline{\widehat{g}}(x-\alpha),
$$

and let $\widetilde{\varphi}(x):=[\hat{\varphi}, \widehat{\varphi}](x)^{1 / 2}$. It is shown in $[\mathbf{5}, \mathbf{6}, \mathbf{9}, \mathbf{1 0}]$ that

$$
\widehat{S}_{\| x}:=\left\{\widehat{f}_{\| x}: f \in S\right\}=\overline{\operatorname{span}}\left\{\widehat{\varphi}_{\| x}: \varphi \in \Phi\right\} .
$$

$\widehat{S}_{\| x}$ is called the fibre space at $x$. The following is [4, Theorem 2.9]. We use the convention that $g(x) / h(x)=0$ for $x \notin \operatorname{supp}(h):=\left\{x \in \mathbb{R}^{d}: h(x) \neq 0\right\}$. 
ThEOREM 2. $([3,4,5])$ Let $S:=\mathcal{S}(\{\varphi\})$, and let $P_{\varphi}$ be the orthogonal projection onto $S$ for $\varphi \in L^{2}\left(\mathbb{R}^{d}\right)$. Then,

$$
\left(P_{\varphi} f\right)_{\| x}=\frac{[\widehat{f}, \hat{\varphi}](x)}{[\widehat{\varphi}, \hat{\varphi}](x)} \widehat{\varphi}(x)
$$

for almost every $x \in \mathbb{T}^{d}$.

The spectrum $\sigma(S)$ of $S$ is defined to be $\left\{x \in \mathbb{T}^{d}: \widehat{S}_{\| x} \neq\{0\}\right\}$. Note that the spectrum is defined modulo sets of Lebesgue measure zero. In what follows, all subsets of $\mathbb{R}^{d}$ are considered modulo Lebesgue measure zero sets with occasional exceptions which are clear from the contexts. For a finite $\Phi \subset L^{2}\left(\mathbb{R}^{d}\right)$ and $x \in \mathbb{T}^{d}$, let $G_{\Phi}(x):=([\hat{\varphi}, \widehat{\psi}](x))_{\varphi, \psi \in \Phi}$, a non-negative definite matrix, which we call the Gramian of $\mathcal{S}(\Phi)$ at $x$. The following is [16, Theorem 2.3.6].

THEOREM 3. ([16]) Let $\lambda_{\Phi}^{+}(x)$ be the smallest non-zero eigenvalue of $G_{\Phi}(x)$ and $\Lambda_{\Phi}(x)$ be the largest eigenvalue of $G_{\Phi}(x)$ for $x \in \mathbb{T}^{d}$. Then, $\left\{T_{\alpha} \varphi: \alpha \in \mathbb{Z}^{d}, \varphi \in \Phi\right\}$ is a frame for $\mathcal{S}(\Phi)$ with frame bounds $A$ and $B$ if and only if $A \leqslant \lambda_{\Phi}^{+}(x) \leqslant \Lambda_{\Phi}(x) \leqslant B$ for almost every $x \in \sigma(\mathcal{S}(\Phi))$.

Notice that (1) implies that $\sigma(\mathcal{S}(\Phi))=\left\{x \in \mathbb{T}^{d}: G_{\Phi}(x) \neq 0\right\}$ and that $\operatorname{dim}\left((\mathcal{S}(\Phi))_{\| x}\right)=\operatorname{rank} G_{\Phi}(x)$ for almost every $x \in \mathbb{T}^{d}$. Notice also that the rank of $G_{\Phi}(x)$ is independent of the generator $\Phi$, whereas the eigenvalues of $G_{\Phi}(x)$ are not.

\section{Gramian analysis}

Suppose that a frame multiresolution analysis $\left(\left\{V_{k}\right\}_{k \in \mathbf{Z}}, \varphi\right)$ is given. Then, obviously $V_{0}=\mathcal{S}(\{\varphi\})$. Moreover, we have, by (1),

$$
\sigma\left(V_{0}\right)=\operatorname{supp}(\tilde{\varphi}(\cdot)) .
$$

Let $A$ and $B$ be the frame bounds of the integer translates of $\varphi$. Since $V_{0} \subset V_{1}=D\left(V_{0}\right)$ and since $\left\{D T_{\alpha} \varphi: \alpha \in \mathbb{Z}^{d}\right\}$ is a frame for $V_{1}$, there exists $m \in L^{2}\left(\mathbb{T}^{d}\right)[\mathbf{5}]$ such that

$$
\widehat{\varphi}(x)=m\left(\frac{x}{2}\right) \hat{\varphi}\left(\frac{x}{2}\right) \text { almost every } x \in \mathbb{R}^{d} .
$$

This $m$ is not unique, since $\left\{D T_{\alpha} \varphi: \alpha \in \mathbb{Z}^{d}\right\}$ is only assumed to be a frame, not necessarily a Riesz basis. Notice that each $\alpha \in \mathbb{Z}^{d}$ can be written uniquely as $\alpha=2 \alpha^{\prime}+\beta$ for some $\alpha^{\prime} \in \mathbb{Z}^{d}$ and some

$$
\beta \in P:=\left\{\left(\varepsilon_{1}, \varepsilon_{2}, \cdots, \varepsilon_{d}\right): \varepsilon_{i}=0 \text { or } 1,1 \leqslant i \leqslant d\right\} .
$$

We see that $\left\{D T_{\alpha} \varphi: \alpha \in \mathbb{Z}^{d}\right\}=\left\{T_{\alpha} D T_{\beta} \varphi: \alpha \in \mathbb{Z}^{d}, \beta \in P\right\}$ by noticing that $D T_{y}=T_{(1 / 2) y} D$ for $y \in \mathbb{R}^{d}$. This shows that $V_{1}=\mathcal{S}(\Pi)$, where

$$
\Pi:=\left\{D T_{\beta} \varphi: \beta \in P\right\} .
$$


Note that the integer translates of II form a frame for $V_{1}$. Let $P_{\varphi}$ be the orthogonal projection onto $\mathcal{S}(\{\varphi\})=V_{0}$, and let

$$
\Xi:=\left\{\xi_{\beta}:=\left(I-P_{\varphi}\right)\left(D T_{\beta} \varphi\right), \beta \in P\right\}
$$

Then $I-P_{\varphi}$ is the orthogonal projection onto $W_{0}:=V_{1} \ominus V_{0} . W_{0}$ is shift-invariant by [5, Corollary 3.4]. Since $P_{\varphi}$ commutes with each integer translation operator [5], it is easy to see that $\left\{T_{\alpha} \xi_{\beta}: \alpha \in \mathbb{Z}^{d}, \beta \in P\right\}$ is also a frame for $W_{0}$ with the same frame bounds $A$ and $B$, hence a wavelet set. In particular, $W_{0}=\mathcal{S}(\Xi)$. Theorem 3 implies that

$$
A \leqslant \lambda_{\Xi}^{+}(x) \leqslant \Lambda_{\Xi}(x) \leqslant B \text { almost every } x \in \sigma\left(W_{0}\right),
$$

where $\lambda_{\Xi}^{+}(x)$ and $\Lambda_{\Xi}(x)$ denote the smallest non-zero eigenvalue and the largest eigenvalue of $G_{\Xi}(x)$, respectively. We sharpen these inequalities in Corollary 7 . Following $[1,2,13]$, we say that the frame multiresolution analysis admits a minimal wavelet set if there is a wavelet set consisting of $2^{d}-1$ elements whose integer translates form a frame for $W_{0}$. When $d=1$, the existence problem of a minimal wavelet set is addressed in [2, 13]. Theorem 5 generalises the results in $[2,13]$.

We now perform a finer Gramian analysis. First, we calculate $G_{\Pi}(x)$. Notice that $\left(D T_{\alpha} \varphi\right)^{\wedge}(x)=2^{-d / 2} e^{-\pi i \alpha \cdot x} \widehat{\varphi}(x / 2)$. Hence, for $x \in \mathbb{T}^{d}, \alpha, \beta \in P$,

$$
\begin{aligned}
G_{\Pi}(x)_{\alpha, \beta} & =\left[\left(D T_{\alpha} \varphi\right)^{\wedge},\left(D T_{\beta} \varphi\right)^{\wedge}\right](x) \\
& =2^{-d} \sum_{\gamma \in \mathbf{Z}^{d}} e^{-\pi i \alpha \cdot(x-\gamma)} \hat{\varphi}\left(\frac{x-\gamma}{2}\right) e^{\pi i \beta \cdot(x-\gamma)} \overline{\hat{\varphi}}\left(\frac{x-\gamma}{2}\right) \\
& =2^{-d} e^{-\pi i(\alpha-\beta) \cdot x} \sum_{\gamma^{\prime} \in \mathbf{Z}^{d}} e^{\pi i(\alpha-\beta) \cdot \gamma^{\prime}}\left|\widehat{\varphi}\left(\frac{x-\gamma^{\prime}}{2}\right)\right|^{2} \\
& =2^{-d} e^{-\pi i(\alpha-\beta) \cdot x} \sum_{\delta \in \mathbf{Z}^{d}, \gamma \in P} e^{\pi i(\alpha-\beta) \cdot(2 \delta+\gamma)}\left|\widehat{\varphi}\left(\frac{x-(2 \delta+\gamma)}{2}\right)\right|^{2} \\
& =2^{-d} e^{-\pi i(\alpha-\beta) \cdot x} \sum_{\delta \in \mathbf{Z}^{d}, \gamma \in P} e^{\pi i(\alpha-\beta) \cdot \gamma}\left|\hat{\varphi}\left(\frac{x-\gamma}{2}-\delta\right)\right|^{2} \\
& =2^{-d} \sum_{\gamma \in P} e^{-\pi i(\alpha-\beta) \cdot(x-\gamma)} \tilde{\varphi}\left(\frac{x-\gamma}{2}\right)^{2} \cdot
\end{aligned}
$$

We observe that, for $\alpha \in P$,

$$
\sum_{\beta \in P} e^{\pi i \alpha \cdot \beta}= \begin{cases}2^{d}, & \text { if } \alpha=(0,0, \cdots, 0) \\ 0, & \text { otherwise. }\end{cases}
$$

The following lemma is a slight variation of [3, Lemma 2.33]. Let $G^{t}$ denote the transpose of a matrix or a vector $G$. 
LEMma 4. For $\mu \in P$ and $x \in \mathbb{T}^{d}, a_{\mu}:=\left(e^{-\pi i \rho \cdot(x-\mu)}\right)_{\rho \in P}^{t}$ is an eigenvector of $G_{\Pi}(x)$ with respect to the eigenvalue $\widetilde{\varphi}((x-\mu) / 2)^{2}$.

Proof: The $\alpha$-th element of $G_{\Pi}(x) a_{\mu}$ is

$$
\begin{aligned}
\left(G_{\Pi}(x) a_{\mu}\right)_{\alpha} & =\sum_{\beta \in P}\left(2^{-d} \sum_{\gamma \in P} e^{-\pi i(\alpha-\beta) \cdot(x-\gamma)} \tilde{\varphi}\left(\frac{x-\gamma}{2}\right)^{2}\right) e^{-\pi i \beta \cdot(x-\mu)} \\
& =2^{-d} e^{-\pi i \alpha \cdot(x-\mu)} \sum_{\gamma \in P} \tilde{\varphi}\left(\frac{x-\gamma}{2}\right)^{2} \sum_{\beta \in P} e^{-\pi i(\alpha-\beta) \cdot(\mu-\gamma)} \\
& =e^{-\pi i \alpha \cdot(x-\mu)} \tilde{\varphi}\left(\frac{x-\mu}{2}\right)^{2}
\end{aligned}
$$

by (5).

For $x \in \mathbb{T}^{d}$, define a $2^{d} \times 2^{d}$ unitary matrix $E(x):=2^{-d / 2}\left(e^{-\pi i \alpha \cdot(x-\beta)}\right)_{\alpha, \beta \in P}$ and a diagonal matrix $D(x):=\operatorname{diag}\left(\tilde{\varphi}((x-\gamma) / 2)^{2}\right)_{\gamma \in P}$. Then, the spectral theorem implies that

$$
G_{\Pi}(x)=E(x) D(x) E(x)^{*},
$$

where * denotes the adjoint of a matrix. Hence, (1) implies that

$$
\operatorname{dim}\left(\widehat{V}_{1|| x}\right)=\operatorname{rank} G_{\Pi}(x)=\#\left\{\tilde{\varphi}\left(\frac{x-\gamma}{2}\right)^{2} \neq 0: \gamma \in P\right\}
$$

We also have

$$
\sigma\left(V_{1}\right)=\bigcup_{\gamma \in P} \operatorname{supp}\left(\tilde{\varphi}\left(\frac{\cdot-\gamma}{2}\right)\right)
$$

Note that (3) and the periodisation technique imply that

$$
\tilde{\varphi}(x)^{2}=\sum_{\alpha \in P}\left|m\left(\frac{x-\alpha}{2}\right)\right|^{2} \tilde{\varphi}\left(\frac{x-\alpha}{2}\right)^{2} .
$$

By [5, Corollary 3.4] we have $\widehat{V}_{0 \| x} \oplus \widehat{W}_{0 \| x}=\widehat{V}_{1|| x}$ for almost every $x \in \mathbb{T}^{d}$. In particular,

$$
\operatorname{dim}\left(\widehat{V}_{0 \| x}\right)+\operatorname{dim}\left(\widehat{W}_{0|| x}\right)=\operatorname{dim}\left(\widehat{V}_{1 \| x}\right)
$$

for almost every $x \in \mathbb{T}^{d}$. These facts lead us to:

THEOREM 5. The frame multiresolution analysis admits a minimal wavelet set if and only if

$$
E:=\left\{x \in \mathbb{T}^{d}: \tilde{\varphi}\left(\frac{x-\alpha}{2}\right) \neq 0, m\left(\frac{x-\alpha}{2}\right)=0 \text { for each } \alpha \in P\right\}
$$

is of Lebesgue measure zero. 
Proof: (7), (9) and (10) imply that $E$ consists of those $x \in \mathbb{T}^{d}$ at which $\operatorname{dim}\left(\widehat{V}_{1|| x}\right)$ $=2^{d}, \operatorname{dim}\left(\widehat{V}_{0|| x}\right)=0$ and $\operatorname{dim}\left(\widehat{W}_{0 \| x}\right)=2^{d}$. Hence if $E$ has a positive measure, then the length of $W_{0}$ should be $2^{d}$ by [5, Theorem 3.5]. This proves the necessity. On the other hand, if $E$ is of zero Lebesgue measure, then (10) implies that $\operatorname{dim}\left(\widehat{W}_{0 \| x}\right) \leqslant 2^{d}-1$ almost everywhere. Then, again by [5, Theorem 3.5], the length of $W_{0}$ is less than or equal to $2^{d}-1$. A slight modification of [6, Theorem 3.3] shows that $W_{0}$ has a generator, with $2^{d}-1$ elements, such that the integer translates of the elements of the generator form a frame for $W_{0}$.

An argument similar to the one in the proof of [13, Lemma 3.4] shows that $E$ is independent of the 1-periodic function $m$. We say that a frame multiresolution analysis is degenerate if $E$ has a positive measure. Examples of degenerate and non-degenerate frame multiresolution analyses are found in $[1,2,13]$. In [3], de Boor et al. noted that $V_{1}=\mathcal{S}(\Theta)$, where $\Theta:=\left\{T_{\alpha / 2} \varphi: \alpha \in P\right\}$, if $\operatorname{supp}(\widehat{\varphi})=\mathbb{R}^{d}$. Note that $\varphi \in \Theta$. It is seen easily $([3])$ that

$$
W_{0}=\mathcal{S}\left\{\left(I-P_{\varphi}\right) T_{\alpha / 2} \varphi: \alpha \in P \backslash\{(0,0, \ldots, 0)\}\right\}
$$

if the integer translates of the scaling function form a Riesz basis of $V_{0}$. This generator is minimal since it consists of $2^{d}-1$ elements. Using this generator of $W_{0}$, de Boor, DeVore and Ron constructed interesting new wavelet sets, and recovered some of the previous constructions of wavelet sets. If, however, the frame multiresolution analysis considered is degenerate, then the generator of de Boor et al. is inadequate simply because one more element is required to be a generator for the degenerate frame multiresolution analysis. Since $\Xi$ is a generator of $W_{0}$ whether the frame multiresolution analysis is degenerate or not, it can be used as a starting point of a construction of a frame wavelet set. Recall that once a generator of a shift-invariant space is found, the other generators are essentially given by 1-periodic function-valued matrices and the Gramian of the generator $[5$, Corollary 3.23]. We give a more detailed analysis of $\Xi$.

We calculate the Gramian $G_{\Xi}(x)$ of $W_{0}$ at $x \in \mathbb{T}^{d}$ with respect to $\Xi$.

$$
\begin{aligned}
G_{\Xi}(x)_{\alpha, \beta}= & {\left[\left(D T_{\alpha} \varphi-P_{\varphi} D T_{\alpha} \varphi\right)^{\wedge},\left(D T_{\beta} \varphi-P_{\varphi} D T_{\beta} \varphi\right)^{\wedge}\right](x) } \\
= & G_{\Pi}(x)_{\alpha, \beta}-\left[\left(D T_{\alpha} \varphi\right)^{\wedge},\left(P_{\varphi} D T_{\beta} \varphi\right)^{\wedge}\right](x) \\
& \quad-\left[\left(P_{\varphi} D T_{\alpha} \varphi\right)^{\wedge},\left(D T_{\beta} \varphi\right)^{\wedge}\right](x)+\left[\left(P_{\varphi} D T_{\alpha} \varphi\right)^{\wedge},\left(P_{\varphi} D T_{\beta} \varphi\right)^{\wedge}\right](x) \\
= & G_{\Pi}(x)_{\alpha, \beta}-\left[\left(D T_{\alpha} \varphi\right)^{\wedge},\left(P_{\varphi} D T_{\beta} \varphi\right)^{\wedge}\right](x) .
\end{aligned}
$$

The last equality follows since

$$
\left[\widehat{f},\left(P_{\varphi} g\right)^{\wedge}\right](x)=\left[\left(P_{\varphi} f\right)^{\wedge}, \hat{g}\right](x)=\left[\left(P_{\varphi} f\right)^{\wedge},\left(P_{\varphi} g\right)^{\wedge}\right](x)
$$

for any $f, g \in L^{2}\left(\mathbb{R}^{d}\right), x \in \mathbb{T}^{d}$ by Theorem 2. Temporarily, let

$$
G(x):=\left(\left[\left(D T_{\alpha} \varphi\right)^{\wedge},\left(P_{\varphi} D T_{\beta} \varphi\right)^{\wedge}\right](x)\right)_{\alpha, \beta \in P} .
$$


Then $G_{\Xi}(x)=G_{\Pi}(x)-G(x)$.

$$
\begin{aligned}
& G(x)_{\alpha, \beta}=\left[\left(D T_{\alpha} \varphi\right)^{\wedge}, \frac{\left[\left(D T_{\beta} \varphi\right)^{\wedge}, \hat{\varphi}\right](x)}{\widetilde{\varphi}(x)^{2}} \widehat{\varphi}\right](x) \\
& =\frac{1}{\widetilde{\varphi}(x)^{2}}\left[\left(D T_{\alpha} \varphi\right)^{\wedge}, \widehat{\varphi}\right](x)\left[\widehat{\varphi},\left(D T_{\beta} \varphi\right)^{\wedge}\right](x) \\
& =\frac{1}{2^{d} \widetilde{\varphi}(x)^{2}}\left(\sum_{\gamma \in \mathbf{Z}^{d}} e^{-\pi i \alpha \cdot(x-\gamma)} \widehat{\varphi}\left(\frac{x-\gamma}{2}\right) \bar{\varphi}(x-\gamma)\right) \\
& \times\left(\sum_{\delta \in \mathbf{Z}^{d}} e^{\pi i \beta \cdot(x-\delta)} \overline{\hat{\varphi}}\left(\frac{x-\delta}{2}\right) \hat{\varphi}(x-\delta)\right) \\
& =\frac{1}{2^{d} \widetilde{\varphi}(x)^{2}}\left(\sum_{\gamma \in \mathbf{Z}^{d}} e^{-\pi i \alpha \cdot(x-\gamma)} \bar{m}\left(\frac{x-\gamma}{2}\right)\left|\hat{\varphi}\left(\frac{x-\gamma}{2}\right)\right|^{2}\right) \\
& \times\left(\sum_{\delta \in \mathbf{Z}^{d}} e^{\pi i \beta \cdot(x-\delta)} m\left(\frac{x-\delta}{2}\right)\left|\hat{\varphi}\left(\frac{x-\delta}{2}\right)\right|^{2}\right) \\
& =\frac{1}{2^{d} \widetilde{\varphi}(x)^{2}}\left(\sum_{\gamma \in P} e^{-\pi i \alpha \cdot(x-\gamma)} \bar{m}\left(\frac{x-\gamma}{2}\right) \tilde{\varphi}\left(\frac{x-\gamma}{2}\right)^{2}\right) \\
& \times\left(\sum_{\delta \in P} e^{\pi i \beta \cdot(x-\delta)} m\left(\frac{x-\delta}{2}\right) \tilde{\varphi}\left(\frac{x-\delta}{2}\right)^{2}\right),
\end{aligned}
$$

where we used the periodisation technique in the last equality, and (3) in the penultimate equality. Define a $2^{d}$-dimensional column vector

$$
M(x):=\left(\bar{m}\left(\frac{x-\alpha}{2}\right) \widetilde{\varphi}\left(\frac{x-\alpha}{2}\right)^{2}\right)_{\alpha \in P}^{t}
$$

Then,

$$
\widetilde{\varphi}(x)^{2} G(x)_{\alpha, \beta}=\left(\alpha^{t h} \text {-element of } E(x) M(x)\right) \cdot\left(\beta^{\text {th }} \text {-element of } \bar{E}(x) \bar{M}(x)\right) .
$$

Hence

$$
\begin{aligned}
G(x) & =\frac{1}{\widetilde{\varphi}(x)^{2}}(E(x) M(x)) \times(\bar{E}(x) \bar{M}(x))^{t} \\
& =\frac{1}{\widetilde{\varphi}(x)^{2}} E(x) M(x) M(x)^{*} E(x)^{*} .
\end{aligned}
$$

This shows that

$$
\begin{aligned}
G_{\equiv}(x) & =G_{\Pi}(x)-G(x) \\
& =E(x)\left(D(x)-\frac{1}{\widetilde{\varphi}(x)^{2}} M(x) M(x)^{*}\right) E(x)^{*} .
\end{aligned}
$$

Hence $G_{\Xi}(x)$ is unitarily equivalent to $D(x)-1 / \widetilde{\varphi}(x)^{2} M(x) M(x)^{*}$. In particular, the eigenvalues of the two matrices coincide. 
Let $\lambda_{\Pi}^{+}(x)$ be the smallest non-zero eigenvalue of $G_{\Pi}(x)$, and $\Lambda_{\Pi}(x)$ the largest eigenvalue of $G_{\Pi}(x)$. Then, Theorem 3 implies that

$$
A \leqslant \lambda_{\Pi}^{+}(x) \leqslant \Lambda_{\Pi}(x) \leqslant B \text { almost every } x \in \sigma\left(V_{0}\right) .
$$

We now characterise the eigenvalues of $G_{\Xi}(x)$. If $\tilde{\varphi}(x)=0$, that is, if $x \notin \sigma\left(V_{0}\right)$, then $G_{\equiv}(x)=G_{\Pi}(x)$. Hence, we concentrate the $x \in \sigma\left(V_{0}\right)$.

THEOREM 6. For $x \in \sigma\left(V_{0}\right)$, the following hold:

(1) 0 is an eigenvalue of $G_{\Xi}(x)$;

(2) Suppose that $\tilde{\varphi}((x-\alpha) / 2)^{2} \neq 0, \alpha \in P$. Then, $\tilde{\varphi}((x-\alpha) / 2)^{2}$ is an eigenvalue of $G_{\Xi}(x)$ if and only if

$$
\sum_{\beta \in I_{\alpha}} m((x-\beta) / 2)=0
$$

where $I_{\alpha}:=\left\{\beta \in P: \widetilde{\varphi}((x-\beta) / 2)^{2}=\widetilde{\varphi}((x-\alpha) / 2)^{2}\right\}$.

(3) Let $\lambda \in \mathbb{R} \backslash\left(\left\{\tilde{\varphi}((x-\gamma) / 2)^{2}: \gamma \in P\right\} \cup\{0\}\right)$. Then, $\lambda$ is an eigenvalue of $G_{\Xi}(x)$ if and only if it satisfies the following equation:

$$
\begin{aligned}
\sum_{\gamma \in P}\left|m\left(\frac{x-\gamma}{2}\right)\right|^{2} \tilde{\varphi}\left(\frac{x-\gamma}{2}\right)^{2}=\sum_{\gamma \in P} \frac{\tilde{\varphi}((x-\gamma) / 2)^{2}}{\tilde{\varphi}((x-\gamma) / 2)^{2}-\lambda} \\
\times\left|m\left(\frac{x-\gamma}{2}\right)\right|^{2} \tilde{\varphi}\left(\frac{x-\gamma}{2}\right)^{2} .
\end{aligned}
$$

Proof: For notational convenience, let us define $c:=\widetilde{\varphi}(x)^{2}, D:=D(x), M$ $:=M(x), m_{\gamma}:=m((x-\gamma) / 2)$ and $a_{\gamma}:=\tilde{\varphi}((x-\gamma) / 2)^{2}, \gamma \in P$. Then, $G_{\Xi}(x)$ is unitarily equivalent to $D-1 / c M M^{*}$. We also let $E_{\beta}, \beta \in P$, to denote the $\beta$-th standard orthonormal vector of $\mathbb{C}^{2^{d}}$.

(1) This can be seen by (10) if we recall that $\operatorname{rank} G_{\Xi}(x)=\operatorname{dim}\left(\widehat{W}_{0 \| x}\right)$. Or this can be seen directly as follows. Define $Y:=\left(\bar{m}_{\alpha}\right)_{\alpha \in P}^{t} Y \neq 0$ by (9). Then, $D Y=M$ and $\langle Y, M\rangle=c$ by (3). Hence, $\left(D-1 / c M M^{*}\right) Y=D Y-(\langle Y, M\rangle / c) M=0$. This shows that 0 is an eigenvalue of $G_{\Xi}(x)$.

(2) Suppose that $a_{\alpha}$ is a non-zero eigenvalue of $G_{\Xi}(x)$. Then, there exists a nonzero vector $X$ such that $\left(D-1 / c M M^{*}\right) X=a_{\alpha} X$. Hence $\left(D-a_{\alpha} I\right) X=(\langle X, M\rangle / c) M$. If $\langle X, M\rangle \neq 0$, then (13) follows by comparing $\beta$-th row of the previous equation for each $\beta \in I_{\alpha}$. If, on the contrary, $\langle X, M\rangle=0$, then the previous equation shows that $X$ is an eigenvector of $D$ with respect to the eigenvalue $a_{\alpha}$. Hence we may assume that $X=\sum_{\beta \in I_{\alpha}} E_{\beta}$. Then $0=\langle X, M\rangle=a_{\alpha} \sum_{\beta \in I_{\alpha}} \bar{m}_{\beta}$, which implies (13). Suppose now that (13) 
holds. A direct calculation shows that $X:=\sum_{\beta \in I_{\alpha}} E_{\beta}$ is an eigenvector of $D-1 / c M M^{*}$ with respect to the eigenvalue $a_{\alpha}$.

(3) Suppose that (14) holds for $\lambda \neq 0$ and $\lambda \neq a_{\alpha}, \alpha \in P$. Let $X:=\left(a_{\alpha} \bar{m}_{\alpha} /\left(a_{\alpha}\right.\right.$ $-\lambda))_{\alpha \in P}^{t}$. Then, $(D-\lambda I) X=M$ and $\langle X, M\rangle=c$. This shows that $\left(D-1 / c M M^{*}\right) X$ $=\lambda X$. Since $x \in \sigma\left(V_{0}\right), X$ is a non-zero vector by (9). Hence $\lambda$ is an eigenvalue of $G_{\Xi}(x)$. Suppose, on the other hand, that $\lambda$ is an eigenvalue of $D-1 / c M M^{*}$ with an eigenvector $X$. Then, $X=(\langle X, M\rangle / c) \operatorname{diag}\left(1 /\left(a_{\alpha}-\lambda\right)\right)_{\alpha \in P} M$. Since $X$ is a nonzero vector, $\langle X, M\rangle \neq 0$. Left-multiplying $c M^{*}$ on both sides of the equation yields $c\langle X, M\rangle=\langle X, M\rangle \sum_{\alpha \in P} a_{\alpha}^{2}\left|m_{\alpha}\right|^{2} /\left(a_{\alpha}-\lambda\right)$. Hence, $\sum_{\alpha \in P} a_{\alpha}^{2}\left|m_{\alpha}\right|^{2}=c=\sum_{\alpha \in P} a_{\alpha}\left|m_{\alpha}\right|^{2}$ by (9).

COROLlary 7. For almost every $x \in \sigma\left(W_{0}\right)$,

$$
A \leqslant \lambda_{\Pi}^{+}(x) \leqslant \lambda_{\Xi}^{+}(x) \leqslant \Lambda_{\Xi}(x) \leqslant \Lambda_{\Pi}(x) \leqslant B .
$$

Proof: Suppose that $x \in \sigma\left(W_{0}\right) \backslash \sigma\left(V_{0}\right)$. Then, $x \in \sigma\left(V_{1}\right)$ by (10). Since $x \in$ $\sigma\left(V_{1}\right) \backslash \sigma\left(V_{0}\right), G_{\Xi}(x)=G_{\Pi}(x)$. Hence the inequalities hold trivially for this $x$. Suppose that $x \in \sigma\left(W_{0}\right) \cap \sigma\left(V_{0}\right)$. It is enough to show that if $\lambda$ is a non-zero eigenvalue of $G_{\Xi}(x)$ different from $a_{\alpha}$ for each $\alpha \in P$, then $\lambda_{\Pi}^{+}(x) \leqslant \lambda \leqslant \Lambda(x)$. By Theorem 6 , (14) holds for such $\lambda$. Let $Q:=\left\{\alpha \in P: a_{\alpha}>0\right\}$. Then, (14) can be written as

$$
\sum_{\alpha \in Q}\left|m_{\alpha}\right|^{2} a_{\alpha}=\sum_{\alpha \in Q} \frac{a_{\alpha}}{a_{\alpha}-\lambda}\left|m_{\alpha}\right|^{2} a_{\alpha} .
$$

The left-hand side of (15) is not 0 by (9) since $x \in \sigma\left(V_{0}\right)$. Theorem 3 and Lemma 4 imply that $A \leqslant \lambda_{\Pi}^{+}(x) \leqslant a_{\alpha} \leqslant \Lambda_{\Pi}(x) \leqslant B$ for $\alpha \in Q$. If $\lambda<\lambda_{\Pi}^{+}(x)$, then $a_{\alpha} /\left(a_{\alpha}-\lambda\right)>1$ for $\alpha \in Q$. This shows that (15) is impossible to hold. If $\lambda>\Lambda_{\Pi}(x)$, then $a_{\alpha} /\left(a_{\alpha}-\lambda\right)<0$ for $\alpha \in Q$. Again, (15) is impossible to hold.

\section{REFERENCES}

[1] J.J. Benedetto and $\mathrm{S}$. Li, "The theory of multiresolution analysis frames and applications to filter banks', Appl. Comput. Harmon. Anal. 5 (1998), 398-427.

[2] J.J. Benedetto and O.M. Treiber, Wavelet frames: multiresolution analysis and extension principle, Applied and Numerical Harmonic Analysis (Birkhaüser, Boston, 2000).

[3] C. de Boor, R. DeVore and A. Ron, 'On the construction of multivariate (pre)wavelets', Constr. Approx. 9 (1993), 123-166.

[4] C. de Boor, R. DeVore and A. Ron, 'Approximations from shift-invariant subspaces of $L^{2}\left(\mathbb{R}^{d}\right)$ ', Trans. Amer. Math. Soc. 341 (1994), 787-806.

[5] C. de Boor, R. DeVore and A. Ron, 'The structure of finitely generated shift-invariant spaces in $L_{2}\left(\mathbb{R}^{d}\right)$ ', J. Funct. Anal. 119 (1994), 37-78.

[6] M. Bownik, 'The structure of shift-invariant subspaces of $L^{2}\left(\mathbb{R}^{n}\right)$ ', J. Funct. Anal. 177 (2000), 282-309.

[7] I. Daubechies, Ten lectures on wavelets (SIAM, Philadelphia, 1992). 
[8] C. Heil and D. Walnut, 'Continuous and discrete wavelet transforms', SIAM Rev. 31 (1989), 628-666.

[9] H. Helson, Lectures on invariant subspaces (Academic Press, New York, 1964).

[10] R.-Q. Jia, 'Shift-invariant spaces and linear operator equations', Israel J. Math. 103 (1998), 258-288.

[11] R.-Q. Jia and C.A. Micchelli, 'Using the refinement equation for the construction of pre-wavelets, II: powers of two', in Curves and Surfaces, (P. J. Laurent, Le Méhauté and L. Schumaker, Editors) (Academic Press, New York), pp. 209-246.

[12] H.O. Kim, R.Y. Kim and J.K. Lim, 'Semi-orthogonal frame wavelets and frame multi-resolution analyses', Bull. Austral. Math. Soc. 65 (2002), 35-44.

[13] H.O. Kim, and J.K. Lim, 'On frame wavelets associated with frame multi-resolution analysis', Appl. Comput. Harmon. Anal. 10 (2001), 61-70.

[14] S. Mallat, 'Multiresolution approximations and wavelets', Trans. Amer. Math. Soc. 315 (1989), 69-88.

[15] Y. Meyer, Wavelets and operators (Cambridge University Press, Cambridge, 1992).

[16] A. Ron and Z. Shen, 'Frames and stable bases for shift-invariant subspaces of $L_{2}\left(\mathbb{R}^{d}\right)$ ', Canad. J. Math. 47 (1995), 1051-1094.

\section{CHiPS}

KAIST

373-1 Guseong-dong, Yuseong-gu

Daejeon 305-701

Republic of Korea

e-mail: jaekun@ftn.kaist.ac.kr 